\title{
EPISÓDIOS HISTÓRICOS POTENCIALMENTE RICOS PARA ATIVIDADES DE ENSINO E APRENDIZAGEM EM MATEMÁTICA
}

\section{POTENTIALLY RICH HISTORICAL EPISODES FOR MATHEMATICAL TEACHING AND LEARNING ACTIVITIES}

\author{
Severino Carlos Gomes ${ }^{1}$ \\ IFRN \\ Rosângela Araújo da Silva ${ }^{2}$ \\ IFRN/UFRN \\ Bernadete Morey ${ }^{3}$ \\ UFRN
}

\section{Resumo}

O presente artigo é parte de um projeto de ensino e pesquisa em andamento que utiliza episódios da história da matemática para promover formas de colaboração não individualistas de aprendizagem por meio do labor conjunto e para despertar/incentivar nos alunos o gosto pela resolução de tarefas não padronizadas com a exploração de novas possibilidades de ensino e aprendizagem. Para o desenvolvimento do projeto, buscamos na Teoria da Objetivação elementos teóricos para a construção de atividades de ensino e aprendizagem e a concepção de labor conjunto como procedimento metodológico de aplicação e desenvolvimento dessas atividades em sala de aula. $\mathrm{O}$ objetivo do presente artigo é apresentar ideias iniciais sobre como destacar um episódio histórico e a partir dele exemplificar atividades e tarefas que podem ser elaboradas e apresentadas aos alunos, assim como destacar as potencialidades didáticas e pedagógicas de cada tarefa ou de um conjunto de tarefas de acordo com a Teoria da Objetivação. Para isso, utilizaremos um vídeo (A Stray Sumerian Tablet) produzido pela Universidade de Cambridge sobre um tablete de 4.200 anos referente à produção de sabão na antiga Mesopotâmia. As atividades serão voltadas para alunos do primeiro ano do ensino médio do Instituto Federal do Rio Grande do Norte (IFRN). Os resultados esperados versarão sobre a aprendizagem de saberes envolvendo a leitura, a compreensão, a interpretação do tablete e pela mobilização de elementos fundamentais para a formação ético-reflexiva desses jovens estudantes.

Palavras-chave: Matemática Mesopotâmica; Ensino de Matemática; História da Matemática; Teoria da Objetivação.

\footnotetext{
${ }^{1}$ severocarlosgomes@gmail.com.

${ }^{2}$ rosangela.silva@ifrn.edu.br.

3 bernadetemorey@gmail.com.
} 
Episódios históricos potencialmente ricos para atividades de ensino e aprendizagem em matemática Severino Carlos Gomes; Rosângela Araújo da Silva; Bernadete Morey

\begin{abstract}
This article is part of an ongoing teaching and research project that uses episodes from the history of mathematics to promote non-individualistic forms of collaborative learning through joint labour and to arouse/encourage students to enjoy solving non-standard tasks exploring new possibilities for teaching and learning. For the development of the project, we sought in the Theory of Objectification theoretical elements for the construction of teaching and learning activities and the conception of joint labor as a methodological procedure for application and development of these activities in the classroom. The aim of this article is to present initial ideas on how to highlight a historical episode and from it exemplify activities and tasks that can be elaborated and presented to students, as well as highlight the didactic and pedagogical potentialities of each task or a set of tasks, according to Theory of Objectification. We will use a video (A Stray Sumerian Tablet) produced by Cambridge University on a 4,200-year-old tablet for soap production in ancient Mesopotamia. The activities will be aimed at first year high school students at the Instituto Federal do Rio Grande do Norte (IFRN). The expected results will be about learning knowledge involving reading, comprehension, interpretation of the tablet and the mobilization of fundamental elements for the ethical-reflective formation of these young students.
\end{abstract}

Keywords: Mesopotamian Mathematics; Teaching of Mathematics; History of Mathematics; Theory of Objectification.

\title{
Introdução
}

O presente artigo é parte de um projeto de pesquisa em andamento sobre a utilização de episódios da história da matemática para promover formas de colaboração não individualistas de aprendizagem por meio do trabalho de labor conjunto e para despertar/incentivar nos alunos o gosto pela resolução de tarefas não padronizadas com a exploração de novas possibilidades de ensino e aprendizagem.

Sob o título: Episódios da História da Matemática e seu uso didático-pedagógico na formação do estudante independente e matematicamente ativo, o projeto de pesquisa em andamento constitui-se das seguintes partes: (1) apresentação do contexto, (2) breve história do IFRN ${ }^{4}$ e seus campi, (3) os cursos do IFRN, (4) o perfil dos alunos que farão parte do projeto, (4) a Teoria da Objetivação como fundamentação teórica, (5) estudar a História da Matemática e sublinhar os episódios potencialmente ricos pedagogicamente, (6) transformação dos episódios em atividades de sala de aula, (7) a intervenção didática, (8) a análise dos dados obtidos e (9) as conclusões da pesquisa.

No trabalho, ora apresentado, consideramos as partes (5) e (6) do projeto com o estudo de episódio da história da matemática da antiga Mesopotâmia e uma proposição

${ }^{4}$ Instituto Federal de Educação, Ciência e Tecnologia do Rio Grande do Norte

Número Especial - IV Seminário Cearense de História da Matemática

Boletim Cearense de Educação e História da Matemática - Volume 07, Número 20, 418 - 426 (2020)

DOI: $10.30938 /$ bocehm.v7i20.2823 
Episódios históricos potencialmente ricos para atividades de ensino e aprendizagem em matemática Severino Carlos Gomes; Rosângela Araújo da Silva; Bernadete Morey

de uso desse episódio em uma atividade de sala de aula. Esse primeiro episódio relacionado consiste em um vídeo, intitulado: A Stray Sumerian Tablet, produzido pela Universidade de Cambridge sobre um tablete de 4.200 anos referente à fabricação de sabão na antiga Mesopotâmia.

Neste sentido, o objetivo do presente artigo é apresentar ideias iniciais sobre como destacar um episódio histórico e a partir dele exemplificar atividades e tarefas que podem ser elaboradas e apresentadas aos alunos, assim como destacar as potencialidades didáticas e pedagógicas de cada tarefa ou de um conjunto de tarefas de acordo com a Teoria da Objetivação de Luis Radford.

Para essas atividades, o público alvo serão estudantes do primeiro ano do ensino médio do IFRN, campus Natal Zona Norte, dos cursos de Eletrônica, Comércio e Informática para internet, enquanto o tema da história da matemática de nossa escolha é a matemática da antiga Mesopotâmia considerando os estudos de Robson (2008) e, principalmente, Høyrup (2002, 2007, 2017).

\section{Estudo da antiga Mesopotâmia no ensino médio}

As ideias iniciais para nosso estudo surgiram a partir de reflexões sobre posturas assumidas por nós mesmos, professores, nossos colegas e, às vezes, por nossos alunos pós-graduandos. Acontece de, ao nos darmos conta das coisas interessantes que poderiam fazer parte de nossa vida profissional envolvendo linguagem bem distinta daquelas a que estamos acostumados, como por exemplo, as línguas mortas: latim, sumério, copta, grego antigo, etc., já estamos na idade adulta e temos a sensação de ser muito tarde para começar do zero.

Tendo constatado isso repetidas vezes em pesquisadores, colegas de trabalho, alunos pós-graduandos e, até mesmo, em graduandos, propusemo-nos a buscar modos de minimizar barreiras que venham a dificultar a entrada num mundo novo por meio do trabalho junto a estudantes mais jovens, isto é, atuando junto a estudantes do ensino médio.

Estamos, então, à procura de atividades para serem desenvolvidas com alunos de ensino médio. Atividades tais que abram espaço para a ousadia característica dos adolescentes e, ao mesmo tempo, permitam que estes desenvolvam habilidades que lhes serão úteis em suas futuras profissões. Aqui estamos focando nossa atenção nas 
Episódios históricos potencialmente ricos para atividades de ensino e aprendizagem em matemática Severino Carlos Gomes; Rosângela Araújo da Silva; Bernadete Morey

habilidades ligadas diretamente à autonomia, à exploração, ao risco, à interpretação de fatos e à busca de subsídios para tomadas de decisões.

Pensamos, então, num estudo histórico-pedagógico em história da matemática antiga cujo objetivo é introduzir alunos de ensino médio em atividades de interpretação de artefatos e linguagens da história antiga, dando ênfase aqueles de viés matemático. Para precisar um pouco mais, diremos que as atividades previstas são diversificadas e centradas na matemática da antiga Mesopotâmia, destacando o estudo dos rudimentos de sua linguagem escrita, de seus sistemas de medida, de seus sistemas de numeração, etc.

Como exposto por Morey e Gomes (2018), o estudo da matemática mesopotâmica antiga se presta muito bem a um trabalho pedagógico nos moldes aqui propostos, pois as fontes disponíveis representam a vida social e econômica em detalhes que permitem a elaboração de atividades originais para a sala de aula.

Vejamos a seguir os fundamentos teóricos para o estudo, a elaboração e a aplicação das atividades.

\section{Labor conjunto nas aulas de matemática}

De caráter sociocultural e semiótico, a Teoria da Objetivação é uma teoria de aprendizagem que atribui à cultura, aos signos, aos artefatos, aos gestos, papel fundamental na tomada de consciência dos objetos do conhecimento (RADFORD, 2006). Além disso, essa teoria defende que as salas de aula não produzem apenas conhecimento, mas também subjetividades. Ou seja, na Teoria da Objetivação, a aprendizagem tanto é um processo de conhecimento quanto um processo de tornar-se. (RADFORD, 2008, 2015).

Com relação à configuração didática de atividades de ensino e aprendizagem, a Teoria da Objetivação considera que toda atividade está associada a uma tarefa e está constituída por uma série de problemas. Nesse sentido, a configuração da atividade deve levar em conta três elementos: 1) condições gerais para execução da atividade, 2) considerações sobre os problemas e 3) considerações sobre a forma de colaboração humana.

Portanto, ao planejar a atividade, primeiramente, o professor deve estar ciente do que já sabem os alunos e envolver, na medida do possível, o uso de artefatos (material concreto, tecnológico, etc.) na atividade. Com relação aos problemas, eles deverão ser interessantes do ponto de vista dos alunos, possibilitar a tomada de consciência dos 
Episódios históricos potencialmente ricos para atividades de ensino e aprendizagem em matemática Severino Carlos Gomes; Rosângela Araújo da Silva; Bernadete Morey

objetos do conhecimento estudados através de níveis profundos de conceituação. Os problemas devem se inter-relacionar de forma que exista uma unidade conceitual e contextual, além de apresentar uma complexidade conceitual crescente.

Com relação ao terceiro elemento, a forma de colaboração humana, refere-se à organização da aula como um espaço para reflexão crítica e de interação profunda entre os estudantes e entre os estudantes e o professor. Ou seja, os problemas propostos na atividade devem exigir compromisso, engajamento, trabalho mútuo de interpretação, reflexão e diálogo entre todos os participantes. (RADFORD, 2014). É o labor conjunto.

No labor conjunto, a aprendizagem consiste em perceber e dar sentido ao conhecimento histórico-cultural de forma ativa e criativa. Também, professores e alunos trabalham juntos, mas eles não fazem as mesmas coisas. A ideia de labor conjunto como postulada pela Teoria da Objetivação traz consigo uma conceituação diferente da divisão do trabalho na atividade de sala de aula e uma conceituação diferente do professor e dos alunos. Nem o professor assume a postura de mediador ou dono do saber, nem os alunos são responsáveis pela sua própria aprendizagem.

A seguir estão as ideias principais de como utilizamos um episódio da história da matemática mesopotâmica para o planejamento de atividades de ensino e aprendizagem em sala de aula com alunos do primeiro ano do ensino médio.

\section{O episódio escolhido e a proposta de atividade}

Em poucas palavras, podemos dizer que, na sala de aula, queremos apresentar aos alunos a sociedade mesopotâmica antiga. No entanto, tal apresentação deverá estar imersa na proposição de atividades desafiadoras que os induzam a conhecer novas forma de organização social, novos (para eles) sistemas de pensamento, novos sistemas de numeração, novos sistemas de medida, novos sistemas de escrita, etc.

Ao mesmo tempo, o trabalho, na sala de aula, deve ser organizado de modo que os alunos nos pequenos grupos entre em contato com o outro na pessoa dos colegas de grupo e com o coletivo de sala de aula. $\mathrm{O}$ teor das atividades inclui assistir vídeos, debates no pequeno grupo e no grande grupo, busca de informação complementares na WEB, resolução de tarefas e problemas, decifrar sistemas simbólicos, etc. 
Episódios históricos potencialmente ricos para atividades de ensino e aprendizagem em matemática Severino Carlos Gomes; Rosângela Araújo da Silva; Bernadete Morey

Para o primeiro encontro, escolhemos um vídeo ${ }^{5}$ (A Stray Sumerian Tablet) produzido pela Universidade de Cambridge sobre um tablete (Figura 1) de 4.200 anos relacionado à fabricação de sabão. $\mathrm{O}$ vídeo tem duração de quase 6 minutos e consiste numa apresentação bastante circunstanciada do tablete pelo professor Nicholas Postgate, membro sênior do Instituto McDonald de Pesquisa Arqueológica de Cambridge. Já providenciamos a colocação de legendas em português.

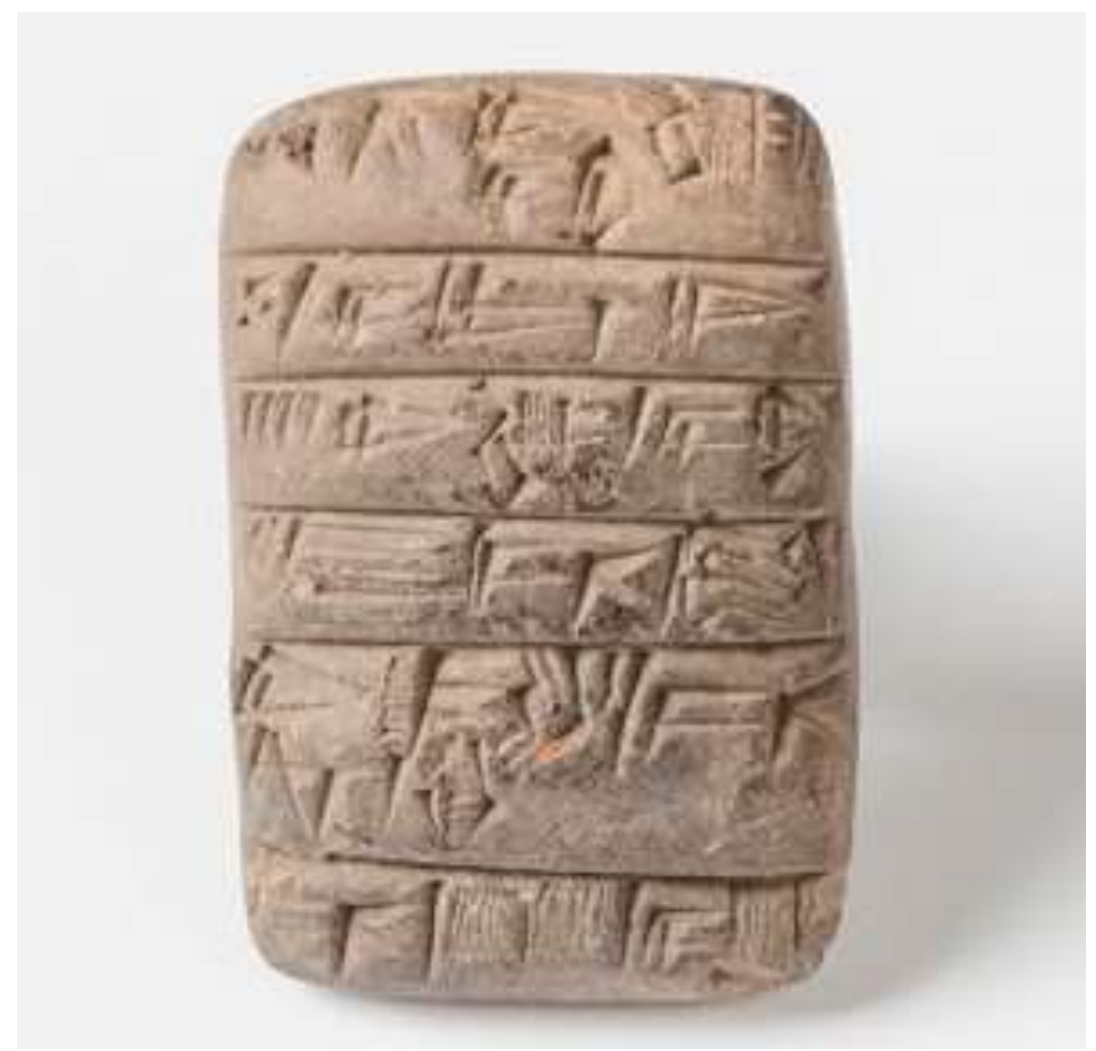

Figura 1 - Tablete MS Doc. 829 (Cambridge Digital Library)

O tablete da Figura 1 contém seis linhas de escrita cuneiforme no idioma sumério e é, aproximadamente, do tamanho de um polegar adulto. Apesar de doado à Biblioteca da Universidade de Cambridge em 1921, somente em 2016 passou a ser estudado durante pesquisa para uma exposição sobre objetos curiosos, realizada como parte do $600^{\circ}$ aniversário da Biblioteca. Esse tablete ficará no centro de nossa atenção enquanto propomos e resolvemos, em labor conjunto, as distintas tarefas relacionadas.

Vale salientar que a escolha para estudo do tablete da Figura 1 se enquadra na ideia de apresentar a sociedade mesopotâmica antiga em seu contexto histórico-cultural,

${ }^{5}$ Acessado em https://www.youtube.com/watch?v=Mm4mXmivuZg3 
Episódios históricos potencialmente ricos para atividades de ensino e aprendizagem em matemática Severino Carlos Gomes; Rosângela Araújo da Silva; Bernadete Morey

visto que "[...] textos e artefatos matemáticos antigos, se quisermos compreendê-los completamente, devem ser vistos à luz de seu contexto histórico-matemático e não devem ser tratados como criações artificiais e independentes...” (ROBSON, 2001, p. 167).

Para essa primeira atividade, seguiremos a seguinte programação: apresentação de professores, alunos e dos objetivos da atividade; (2) exibição do vídeo (A Stray Sumerian Tablet) legendado com duração de 5m32s; (3) discussão sobre o conteúdo do vídeo com a retomada de alguns trechos; (4) dividir os alunos em grupos com no máximo quatro componentes; (5) os grupos receberão uma folha de papel com algumas perguntas baseadas no vídeo para responder. Se necessário, os alunos podem rever trechos do vídeo, desde que seja todo o grupo, para formular e averiguar suas respostas; (6) Terminada a tarefa da folha, um debate geral de toda a classe com apresentação e discussão das respostas dos grupos; (7) atribuição de tarefas para casa retomadas no próximo encontro.

Portanto, como os sujeitos envolvidos no estudo são alunos do primeiro ano dos cursos técnicos/profissionalizantes em Informática, Eletrônica e Comércio, acreditamos que vão se sentir instigados pelas tarefas de:

- Elaboração de tabelas que os mesopotâmicos usavam por meio de breves programas de computador;

- Debater, a partir de leituras prévias, sobre a utilidade de tais tabelas e seu modo de usar;

- Elaborar programa de computador de transformação de unidades de medidas;

- Dado um tablete já decifrado, buscar a confirmação da decifração dada;

- De modo autônomo e sem ajuda das fontes, "decifrar" tabletes fáceis, como, por exemplo, o HS 217a (da multiplicação por 9).

Para essa atividade será pensada para ser realizada em quatro encontros de duas horas-aula cada, em dias letivos consecutivos, ainda no primeiro bimestre de 2020. Os encontros se darão durante o horário regular da turma de alunos participantes. A escolha do horário deve-se ao fato de o desenho do projeto priorizar a participação de todos os alunos independente de sua simpatia ou não pela matemática. 
Episódios históricos potencialmente ricos para atividades de ensino e aprendizagem em matemática Severino Carlos Gomes; Rosângela Araújo da Silva; Bernadete Morey

\section{Considerações finais}

Inúmeros são os desafios encontrados em uma sala de aula de alunos ingressantes no ensino médio no IFRN. Há diversidades emocionais, cognitivas, físicas, psicológicas, sociais, econômicas, culturais, etc. Há aqueles que amam matemática e há também aqueles que a odeiam.

Para enfrentarmos os desafios destas salas heterogêneas, com diversos aspectos que influenciam diretamente na aprendizagem, utilizamos fundamentos teóricos e metodológicos da Teoria da Objetivação, pois essa teoria pressupõe a Educação Matemática "como un esfuerzo político, social, histórico y cultural cuyo fin es la creación de individuos éticos y reflexivos que se posicionan de manera crítica en prácticas matemáticas constituidas histórica y culturalmente." (RADFORD, 2014, p. 135).

Nesse sentido, apresentamos neste texto ideias iniciais de projeto sobre a utilização de episódios da história da matemática para promover formas de colaboração não individualistas de ensino e aprendizagem e despertar nos alunos o gosto pela resolução de atividades de investigação envolvendo matemática antiga. No caso, o planejamento de uma atividade envolvendo um tablete produzido na antiga Mesopotâmia.

Nossa expectativa é que devido à natureza não trivial da atividade, aliada ao labor conjunto como metodologia de ensino postulada pela Teoria da Objetivação, o trabalho em sala de aula venha a promover uma atmosfera na qual os alunos se sintam em pé de igualdade quanto ao desenvolvimento das tarefas. Mas, somente a natureza da atividade não será suficiente para aproximar os alunos entre si, caso as tarefas não tenham um desenho adequado. Felizmente, o desenho de atividades no marco teórico da Teoria da Objetivação leva muito a sério o trabalho em grupos e a cooperação entre os participantes da sala de aula.

Portanto, no estudo do tablete da história da matemática mesopotâmica antiga, além da aprendizagem de saberes envolvendo a leitura, a compreensão, a interpretação do texto cuneiforme, o resultado mais importante para nós será a fusão (ou não) dos grupos de alunos em um coletivo único da sala de aula. Será que o labor conjunto ajudará a reduzir as desigualdades em uma sala de aula extremamente heterogênea? Durante o evento IV SCHM em abril já teremos o que dizer. 
Episódios históricos potencialmente ricos para atividades de ensino e aprendizagem em matemática Severino Carlos Gomes; Rosângela Araújo da Silva; Bernadete Morey

\section{Referências}

HØYRUP, J. Algebra in Cuneiform. Introduction to an Old Babylonian Geometrical Technique. Berlin: PRO BUSINESS digital printing Deutschland GmbH, 2017.

HØYRUP, J. The roles of Mesopotamian bronze age mathematics tool for state formation and administration - Carrier of teachers' professional intellectual autonomy. Educational Studies in Mathematics, 2007, p. 257-271.

HØYRUP, J. Lenghts, widths, surfaces: a portrait of old Babylonian algebra and its kin. New York: Springer-Verlag, 2002.

MOREY, B. B.; GOMES, S. C. Matemática mesopotâmica: história para o professor de matemática. REMATEC, Natal/RN, Ano 13, n. 27, p. 06-23, jan./abr., 2018.

RADFORD, L. Methodological Aspects of the Theory of Objectification. Perspectivas da Educação Matemática, v. 8(18), p. 547-567, 2015.

RADFORD, L. On teachers and students. In: Liljedahl, P.; Nicol, C.; Oesterle, S.; Allan, D. (Eds.). Proceedings... Vancouver, Canada: PME, 2014.

RADFORD, L. The ethics of being and knowing: Towards a cultural theory of learning. In: RADFORD, L.; SCHUBRING, G.; SEEGER, F. (Eds.). Semiotics in mathematics education: Epistemology, history, classroom, and culture. Rotterdam: Sense Publishers, 2008 .

RADFORD, L. Elements of a Cultural Theory of Objectification. Revista Latinoamericana de Investigación en Matemática Educativa: Special Issue on Semiotics, Culture and Mathematical Thinking, p. 103-129, 2006.

ROBSON, E. Mathematics in Ancient Iraq: a social history. New Jersey: Princeton University Press, 2008.

ROBSON, E. Neither Sherlock Holmes nor Babylon: a reassessment of Plimpton 322. Historia Mathematica, n. 28, p. 167-206, 2001. 